\title{
NECROLOGIE
}

\section{Maurice Lombard}

\section{L'homme}

Maurice Lombard, que vient d'enlever une longue et cruelle maladie, aura été le plus doué, le plus brillant historien de notre génération, le seul qui fût incontestablement de la classe d'un Marc Bloch. Il avait tout pour réussir : un gout éperdu de vivre - et de bien vivre -, une intelligence toute de finesse, une érudition sans faille, une écriture de très haute qualité, plus le don de la parole et une vaste connaissance des langues classiques et étrangères. Né à Jemmapes (département de Constantine), le 15 octobre 1904, il avait très tôt appris et pratiqué la langue arabe, ce qui l'avait armé à l'avance pour être l'historien sans égal de la Méditerranée sarrasine. $A$ ce vaste sujet, il avait consacré un ouvrage depuis longtemps achevé, quant à l'essentiel, mais que son souci de la perfection l'empếchait encore de publier. Ce souci de la perfection, sa sensibilité à l'égard de critiques intempestives, ont jeté une ombre permanente sur sa vie laborieuse. Maurice Lombard a, de ce fait, peu publié. Encore ai-je di littéralement lui arracher ses publications, une à une. Aujourd'hui, c'est à ses élèves, à ses amis, à son admirable femme que la tâche incombe de sauver une ceuvre exceptionnelle. Mais je suis sûr de leur succès.

L'ampleur, la qualité de son travail se devinent à la lecture de ses quelques articles, ou devant l'énorme travail cartographique accompli par lui avec une exactitude scrupuleuse qui faisait l'admiration des hommes du métier. Nos lecteurs connaissent sa carte des ressources en bois de la Méditerranée durant les siècles de l'hégémonie musulmane. D'autres cartes existent, ainsi celle, admirable, des esclaves que l'Islam a trouvés de gré ou de force en Europe, en Afrique, et en Asie.

Mais l'homme valait plus encore que l'historien : bienveillant, courtois, fraternel, chaleureux, d'une fidélité sans faille dans ses amitiés. Comment dire son charme, son élégance...? Qui de ses amis ou de ses élèves pourrait l'oublier?

Fernand Braudel. 


\section{Le maittre}

Le désarroi que je ressens à avair perdu Maurice Lombard ne vient pas seulement de l'affection et de la reconnaissance. Il était pour moi - comme pour ses autres élèves - la puissance et la vie. Que la mort ait pu en triompher avant l'âge me cause autant d'indignation et de stupeur que de douleur. Pour moi, comme pour la plupart des jeunes historiens qu'il a marqués de son empreinte, Maurice Lombard est né - paradoxalement - avec l'agrégation d'histoire des années 1950-1954. Pourquoi ne pas l'avouer? Ce fut le souci de la préparation du concours qui nous entraîna dans l'aile secrète et écartée de la Sorbonne où officiaient ceux qui venaient de s'emparer de l'agrégation et que d'aucuns nous peignaient comme des diables prêts à entraîner dans l'enfer d'une histoire hérétique et scandaleuse les proies naïves que nous étions. Fernand Braudel, avec sa malice et sa profonde et intelligente gentillesse qui nous refusait un aliment immédiatement et agrégativement assimilable, afin de mieux conserver notre appétit pour une vraie et succulente histoire, ne nous rassurait pas toujours sur son innocence. Nous le retrouvions chez nous avec la Méditerranée aux eaux toutes fraîches qui nous rebaptisait historiens. Maurice Lombard nous réservait des surprises pour ses sujets du concours. Dans la salle Delamarre, que je ne peux plus évoquer sans qu'il l'emplisse de sa puissante présence, il nous fit le plus beau don possible : il nous convainquit que nous avions eu raison de vouloir être historiens, que l'histoire valait la peine d'être aimée, poursuivie, étreinte, qu'elle existait et qu'elle était belle.

Pourtant il avait consenti - sans effort apparent - à travestir l'histoire des oripeaux de la scolastique traditionnelle. Les Barbares, qui étaient de vrais barbares, avec un corps qu'ils nourrissaient, des vêtements, des outils et des armes qu'ils fabriquaient, qui se souvenaient des steppes et qui ne se demandaient pas s'ils étaient en train ou non de poser les bases de la féodalité, entraient docilement dans les trois parties de la dissertation universitaire, se présentaient dans une introduction, se laissaient voir sous trois aspects successifs, sans être coupés en trois comme on le faisait ailleurs, et se retrouvaient plus compréhensibles mais aussi authentiques dans la conclusion. Maurice Lombard fut un grand professeur jusque dans les servitudes de la technique scolaire.

Donc il nous offrit l'histoire. Avec toutes ses dimensions, avec tout son espace. Les hommes du passé vivaient sur une terre vraie, en suivant des routes qui traversaient des montagnes et des plaines, contournaient des obstacles et cherchaient des cols, des fleuves, des courants. Ils ne savaient pas, pour la plupart, écrire; et pour les connaître il valait mieux interroger les objets qu'ils avaient laissés : un morceau de poterie, un fragment d'épée, un lambeau de tissu. Leurs maisons, leur nourriture, leurs gestes 


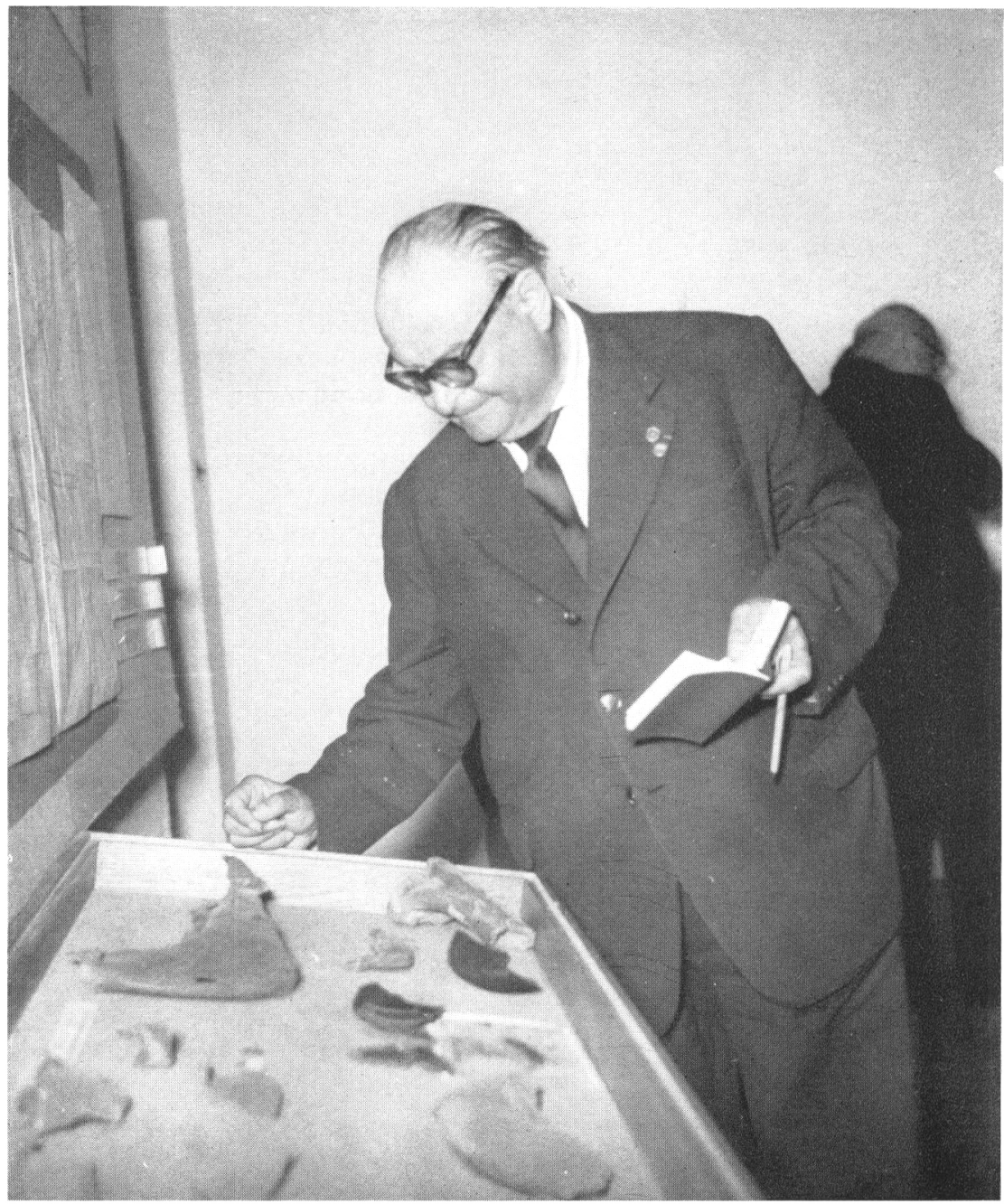

MAIRICE LOMBARD 
les exprimaient mieux que les créations exceptionnelles de leurs élites savantes.

Ils vivaient et surtout ils bougeaient; et la civilisation, les civilisations, se développaient au rythme de leurs déplacements et de leurs contacts. Si Maurice Lombard avait horreur de quelque chose, c'était du clos, du fermé, de l'autarcique, du stérile. Il ne s'intéressait qu'à ce qui créait des richesses, de l'abondance, des surplus.

Vingt-cinq ans après le début de ses études et de ses travaux, nous comprenions ce qui l'avait attiré vers l'Islam. Un monde de refonte et de creuset de civilisations, le grand brassage des techniques, des arts et des hommes, de l'Inde à l'Espagne. Alexandre, la conquête musulmane, les grandes découvertes, voilà quels étaient pour lui les trois grands moments de l'histoire du monde, ceux où le globe entier n'était plus fractionné mais réuni par une large circulation. Le luxe l'hypnotisait, il l'étudiait, comme phénomène économique et social, avec prédilection. Mais s'il était heureux d'y voir la transmutation d'un surplus, il s'inquiétait d'y trouver la stérilisation de richesses et guettait les moments où il rentrerait dans un circuit créateur. Alexandre inondant le monde antique des trésors des temples de l'Orient, les califes envoyant de la Chine à l'Espagne, du Soudan à la Scandinavie, l'or et l'argent enfouis dans les mines et les sables du Turkestan, de la Nubie, du Soudan, voilà ce qui le comblait.

Voilà ce qu'il nous apportait en s'animant toujours lui-même : amples mouvements du buste et des bras qui arrachaient les dirhems et les dinars aux ateliers monétaires de l'Orient et les poussaient jusqu'à l'extrême Occident, jusqu'à nous ; allées et venues au tableau, où sans cesse il dessinait - avec un sens merveilleux de l'essentiel - des routes, un plan, un objet; déroulements de ces grandes et belles cartes qui franchissaient avec lui, le vendredi à 5 heures, la porte de l'École des Hautes Études, où s'engouffrait, avec elles et avec lui, le vent des grands espaces.

Il ne chercha - quand son abandon de l'agrégation entraîna le reflux de la foule — ni à séduire, ni à exploiter le petit troupeau des fidèles qui était resté. Quand il nous demandait d'exposer nos travaux, c'était pour $y$ trouver lui-même, avec une sûreté incomparable, les grandes leçons d'histoire générale que nous n'y avions pas soupçonnées. Il nous laissait avec notre modeste travail enrichi, transfiguré, chargé de toute l'histoire du monde. Presque aucun d'entre nous n'a été un islamisant, mais personne ne nous a mieux éclairés et instruits que lui sur notre domaine propre.

Depuis quelques années un mal mystérieux - qui était l'affreuse maladie - ralentissait le débit de la source. La corne d'abondance ne versait plus à pleins bords. 


\section{A NNALES}

Il jaut bien, face à quelques articles - si beaux soient-ils -, en attendant qu'on arrache à ses papiers leurs trésors enfouis, que nous, qui lui devons tant, portions un témoignage. Il n'était ni impuissant, ni thésaurisateur. Mais il croyait à la nécessité des lentes maturations. Il a cru que le temps lui serait laissé de la récolte et de la mise en circulation. Il n'attendait que cela. Si sa mort me paraît un scandale, c'est qu'il a été l'historien le plus riche mais aussi le plus généreux que j'aie connu et que, s'il a tout donné à ses auditeurs, il n'a pas eu le temps de tout donner à ses lecteurs.

J ACQUeS Le Goff. 